\title{
New applications of CARLOMAT
}

\author{
Karol Kołodziej ${ }^{* \dagger}$ \\ University of Silesia \\ E-mail: karol.kolodziej@us.edu.pl
}

\begin{abstract}
Modifications of CARLOMAT, a program for automatic computation of the lowest order cross sections of multiparticle reactions, that include an abridgement of the phase space integration routine, an interface to parton density functions, improvement of the color matrix computation, supplementation of the Cabibbo-Kobayashi-Maskawa mixing in the quark sector, implementation of effective models such as scalar electrodynamics, the Wtb interaction with operators of dimension up to 5 and a general top-Higgs coupling, are discussed. The modifications, together with recent developments concerning mainly description of the $e^{+} e^{-}$annihilation into hadrons at low energies, broaden the spectrum of possible applications of the program.
\end{abstract}

Loops and Legs in Quantum Field Theory - LL2014,

27 April 2014 - 02 May 2014

Weimar, Germany

\footnotetext{
* Speaker.

$\dagger$ This project was supported in part with financial resources of the Polish National Science Centre (NCN) under grant decision number DEC-2011/03/B/ST6/01615.
} 


\section{Introduction}

CARLOMAT [1] is a program for automatic computation of the lowest order cross sections of multiparticle reactions, dedicated mainly to the description of the processes of production and decay of heavy particles, e.g. top quarks, Higgs boson, or electroweak gauge bosons.

Substantial modifications with respect to version 1 of the program that will be briefly discussed in the subsequent sections of the present lecture include:

- generation of a single phase space parameterization for the Feynman diagrams of the same topology,

- an interface to parton density functions (PDFs),

- improvement of the color matrix computation,

- the Cabibbo-Kobayashi-Maskawa (CKM) mixing in the quark sector,

- effective models, including scalar electrodynamics, the Wtb interaction with operators of dimension up to 5 and a general top-Higgs coupling.

Version 2 of CARLOMAT was released in summer 2013 and the writeup was published in the beginning of 2014 [2].

\section{Phase space integration}

The number of peaks in the squared matrix element usually by far exceeds the number of independent variables in a single parameterization of the phase space integration element. Therefore, the phase space integration in CARLOMAT is performed with the use of the multichannel Monte Carlo (MC) approach. In version 1 of the program, a separate phase space parameterization is generated for each Feynman diagram and the peaks of the corresponding amplitude, which arise if any Feynman propagator approaches its minimum, are smoothed with appropriate mappings of the integration variables. The parameterizations are then automatically combined in a single multichannel phase space integration routine.

However, the Feynman diagrams of the same topology may differ from each other only in propagators of the internal particles. This means that the integration limits of all the invariants, that are uniquely defined for each topology in terms of subsets of four momenta of the final state particles, are the same. Also the Lorentz boosts of four momenta, which are randomly generated in the relative centre of mass system of a given subset of particles, to the centre of mass system are the same. Thus, both the integration limits and boosts can be written only once for all the diagrams of the same topology. The phase space integration routine in CARLOMAT_V2. 0 becomes shorter and the compilation time is reduced by a factor of 4-5 for multiparticle reactions, compared to the previous version of the program. 


\section{Hadron-hadron collisions, color matrix and CKM mixing}

Interfaces to MSTW [3] and CTEQ6 [4] PDFs are added in the MC computation part of the program. The user should choose if she/he wants to calculate the cross section of the hard scattering process at the fixed centre of mass energy, or to fold it with the PDFs, treating the initial state particles as partons of either the $p \bar{p}$ or $p p$ scattering.

Computation of the color matrix in CARLOMAT_V2.0 is performed as a separate stage, that is automatically executed just after the code generation and only the nonzero elements are transferred to the MC program. A subroutine colsqkk that computes the reduced color matrix is divided into smaller subroutines of the user controlled size which allows to compute much larger color matrices and speeds up the compilation process.

The CKM mixing in the quark sector is implemented in the program. However, the complex phase of the CKM matrix can be easily incorporated, as the $W$ boson coupling to fermions that always multiplies $V_{i j}$ is complex anyway. If the CKM mixing is included then the number of Feynman diagrams of hadronic reactions grows substantially. For the sake of simplicity, only the magnitudes of the CKM matrix elements $V_{i j}$ [5] are taken into account. Therefore, as the inclusion of the CKM mixing would be an unnecessary complication for many applications, it can be either switched on or off.

\section{Anomalous $W t b$ and top-Higgs Yukawa couplings}

The effective Lagrangian of the $W t b$ interaction containing operators of dimension four and five that is implemented in the current version of the program has the following form [6]:

$$
\begin{aligned}
L_{W t b} & =\frac{g}{\sqrt{2}} V_{t b}\left[W_{\mu}^{-} \bar{b} \gamma^{\mu}\left(f_{1}^{L} P_{L}+f_{1}^{R} P_{R}\right) t-\frac{1}{m_{W}} \partial_{\nu} W_{\mu}^{-} \bar{b} \sigma^{\mu \nu}\left(f_{2}^{L} P_{L}+f_{2}^{R} P_{R}\right) t\right] \\
& +\frac{g}{\sqrt{2}} V_{t b}^{*}\left[W_{\mu}^{+} \bar{t} \gamma^{\mu}\left(\bar{f}_{1}^{L} P_{L}+\bar{f}_{1}^{R} P_{R}\right) b-\frac{1}{m_{W}} \partial_{\nu} W_{\mu}^{+} \bar{t} \sigma^{\mu v}\left(\bar{f}_{2}^{L} P_{L}+\bar{f}_{2}^{R} P_{R}\right) b\right] .
\end{aligned}
$$

The couplings $f_{i}^{L}, f_{i}^{R}, \bar{f}_{i}^{L}, \bar{f}_{i}^{R}, i=1,2$, can be complex in general. For a detailed explanation of the notation used in Eq. (4.1) see [7]. In the Standard Model (SM), one has $f_{1}^{L}=\bar{f}_{1}^{L}=1$, while other couplings are equal to zero. If $\mathrm{CP}$ is conserved then the following relationships hold:

$$
\bar{f}_{1}^{R^{*}}=f_{1}^{R}, \quad \bar{f}_{1}^{L^{*}}=f_{1}^{L}, \quad \bar{f}_{2}^{R^{*}}=f_{2}^{L}, \quad \bar{f}_{2}^{L^{*}}=f_{2}^{R} .
$$

In order to avoid on-shell poles, masses in the Feynman propagators of unstable particles are substituted by:

$$
m_{b}^{2} \rightarrow m_{b}^{2}-i m_{b} \Gamma_{b}, \quad b=Z, W, h, \quad m_{t} \rightarrow \sqrt{m_{t}^{2}-i m_{t} \Gamma_{t}}
$$

in the $s-, t$ - and $u$-channel. The top quark width $\Gamma_{t}$ in (4.3) is calculated anew every time the form factors $f_{i}^{L}, f_{i}^{R}, \bar{f}_{i}^{L}$ and $\bar{f}_{i}^{R}, i=1,2$, are changed. For CP-odd choices of the couplings, the widths of the top quark $\Gamma_{t}$ and the width of the antitop quark $\Gamma_{\bar{t}}$ calculated using Lagrangian (4.1) differ from each other. Therefore both widths are calculated and the following rule is applied in the $s$ channel top quark propagators: $m_{t} \rightarrow \sqrt{m_{t}^{2}-i m_{t} \Gamma_{t}}$ is used if the propagator goes into $W^{+} b$ and 


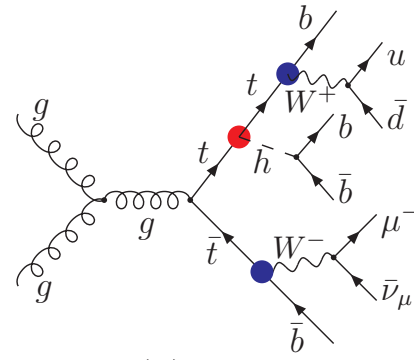

(a)

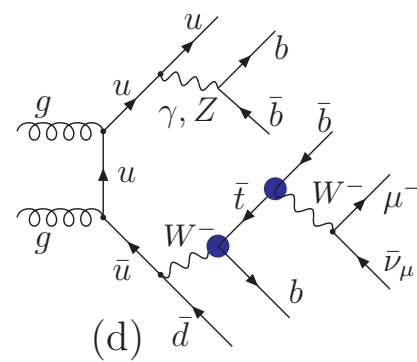

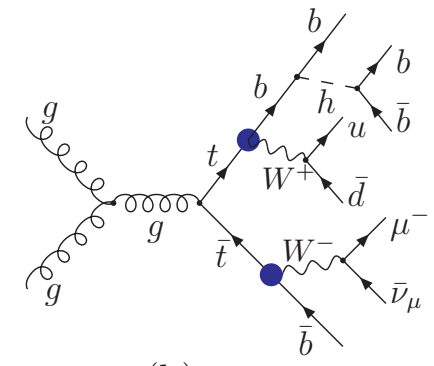

(b)

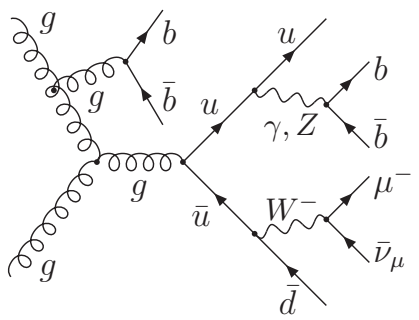

(e)

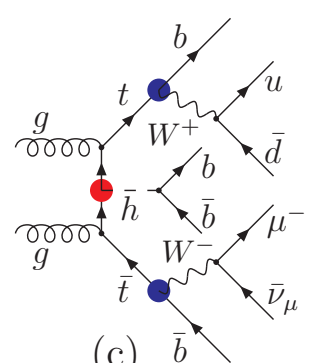

(c) $\bar{b}$

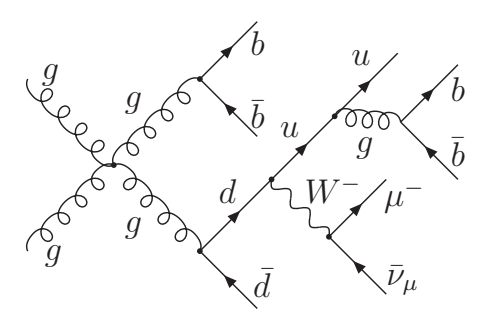

(f)

Figure 1: Examples of the leading order Feynman diagrams of reaction (4.5).

$m_{t} \rightarrow \sqrt{m_{t}^{2}-i m_{t} \Gamma_{\bar{t}}}$ is used if the propagator goes into $W^{-} \bar{b}$ [7]. The rule does not work for the propagators in $t$ - or $u$-channels, but the actual value of the width should not play much of a role there. The prescription was to some extent justified using unitarity arguments in [7], but its field theoretical justification would actually require the calculation of higher order corrections with the nonrenormalizable Lagrangian (4.1).

The most general Lagrangian of $t \bar{t} h$ interaction including corrections from dimension-six operators that has been implemented in the program has the following form [8]:

$$
\mathscr{L}_{t \bar{t} h}=-g_{t \bar{t} h} \bar{t}\left(f+i f^{\prime} \gamma_{5}\right) t h .
$$

The couplings $f$ and $f^{\prime}$ in (4.4) that describe the scalar and pseudoscalar departures, respectively, from a purely scalar top-Higgs Yukawa coupling $g_{t \bar{t} h}$ of SM which is reproduced for $f=1$ and $f^{\prime}=0$, are assumed to be real.

In order to illustrate the relevance of automation let us consider the reaction

$$
g g \rightarrow b u \bar{d} \bar{b} \mu^{-} \bar{v}_{\mu} b \bar{b}
$$

which is a dominant partonic subprocess of associated production of the top quark pair and Higgs boson at the LHC. Examples of the leading order Feynman diagrams of reaction (4.5) are shown in Figure 1, where red (blue) blobs indicate the top-Higgs $(W t b)$ coupling. There are 67300 diagrams in the leading order of the SM, in the unitary gauge, neglecting masses smaller than the $b$-quark mass and the CKM mixing. That big number of the diagrams practically excludes a possibility of implementing couplings of Eqs. (4.1) or (4.4) in the matrix element of (4.5) by hand.

The effects of the anomalous top-Higgs Yukawa coupling on different observables in the process of $t \bar{t} H$ production at LHC calculated with CARLOMAT_V2.0 were illustrated in [9]. The program was also used to study effects of the anomalous $W t b$ coupling on the process of top quark pair production in $p \bar{p}$ collisions at the Tevatron [10] and in $p p$ collisions at the LHC [7]. 


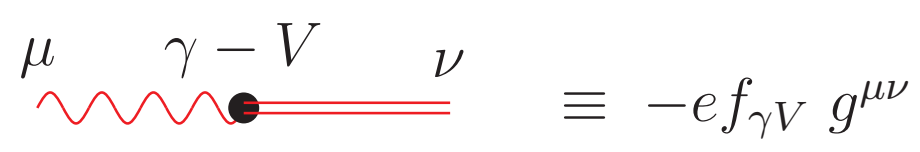

Figure 2: A diagram representing mixing of the photon with neutral vector mesons.

\section{5. $e^{+} e^{-}$annihilation to hadrons at low energies}

The knowledge of the energy dependence of the cross section of $e^{+} e^{-}$annihilation into hadrons, $\sigma_{e^{+} e^{-} \rightarrow \text { hadrons }}(s)$, allows to determine, through dispersion relations, the hadronic contributions to the vacuum polarization which are necessary for better precision of theoretical predictions for the muon anomalous magnetic moment and evolution of the fine structure constant from the Thomson limit to high energy scales.

Below the $J / \psi$ threshold, $\sigma_{e^{+} e^{-} \rightarrow \text { hadrons }}(s)$ must be measured, either by the initial beam energy scan or with the use of a radiative return method [11]. At low energies, the hadronic final states consist mostly of pions, accompanied by one or more photons. The simplest theoretical framework that allows to describe effectively the low energetic electromagnetic (EM) interaction of charged pions is scalar electrodynamics (sQED). At low energies, $\pi^{ \pm}$can be treated as point like particles represented by a complex scalar field $\varphi$. The $U(1)$ gauge invariant Lagrangian of sQED implemented in CARLOMAT has the following form, see e.g. [12]:

$$
\mathscr{L}_{\pi}^{\mathrm{sQED}}=\partial_{\mu} \varphi\left(\partial^{\mu} \varphi\right)^{*}-m_{\pi}^{2} \varphi \varphi^{*}-i e\left(\varphi^{*} \partial_{\mu} \varphi-\varphi \partial_{\mu} \varphi^{*}\right) A^{\mu}+e^{2} g_{\mu \nu} \varphi \varphi^{*} A^{\mu} A^{v} .
$$

The bound state nature of the charged pion can be taken into account by the substitutions:

$$
e \rightarrow e F_{\pi}\left(q^{2}\right), \quad e^{2} \rightarrow e^{2}\left|F_{\pi}\left(q^{2}\right)\right|^{2}
$$

where $F_{\pi}\left(q^{2}\right)$ is the charged pion form factor that has not been implemented in the program yet.

Simulation of processes involving the EM interaction of nucleons are also possible with the most recent version of CARLOMAT. Due to the fact, that the EM current of spin 1/2 nucleons has the form

$$
J^{\mu}=e \bar{N}\left(p^{\prime}\right)\left[\gamma^{\mu} F_{1}\left(Q^{2}\right)+\frac{i}{2 m_{N}} \sigma^{\mu v} q_{v} F_{2}\left(Q^{2}\right)\right] N(p),
$$

which is very similar to the form of the $t b$ current in $L_{W t b}$ of Eq. (4.1), its implementation in the program was straightforward. Form factors $F_{1}\left(Q^{2}\right)$ and $F_{2}\left(Q^{2}\right)$, where $Q^{2}=-\left(p^{\prime}-p\right)^{2}$, have been adopted from PHOKARA [13].

\section{Outlook}

Work on implementation of the Feynman rules of the Resonance Chiral Perturbation Theory provided by Fred Jegerlehner [14] is ongoing. Implementation of new triple and quartic vertices is straightforward. Just several new subroutines for the calculation of the new Lorentz tensor structures that arise in the model must be written and tested. However, the implementation of the particle mixing, as illustrated in Figure 2, is more challenging. It requires substantial changes in the code generating part of the program. 


\section{References}

[1] K. Kołodziej, Comput. Phys. Commun. 180 (2009) 1671 [arXiv:0903.3334];

K. Kołodziej, Acta Phys. Polon. B42 (2011) 2477 [arXiv:1204.5099].

[2] K. Kołodziej, Comput. Phys. Commun. 185 (2014) 323 [arXiv:1305.5096].

[3] A.D. Martin, W.J. Stirling, R. S. Thorne, G. Watt, Eur. Phys. J. C63 (2009) 189 [arXiv:0901.0002].

[4] J. Pumplin et al., JHEP 07 (2002) 012 [hep-ph/0201195].

[5] J. Beringer et al. (Particle Data Group), Phys. Rev. D86 (2012) 010001.

[6] G.L. Kane, G.A. Ladinsky, C.-P. Yuan, Phys. Rev. D45 (1992) 124.

[7] K. Kołodziej, Acta Phys. Pol. B44 (2013) 1775 [arXiv:1212.6733].

[8] J.A. Aguilar-Saavedra, Nucl. Phys. B821 (2009) 215 [arXiv:0904.2387].

[9] K. Kołodziej, Journal of High Energy Physics 07 (2013) 083 [arXiv:1303.4962].

[10] K. Kołodziej, Phys. Lett. B710 (2012), 671 [arXiv:1110.2103].

[11] Min-Shih Chen and P. M. Zerwas, Phys. Rev. D11 (1975) 58.

[12] F. Jegerlehner, The Anomalous Magnetic Moment of the Muon, Springer, Nov. 2007, ISBN 9783540726333.

[13] S. Tracz, H. Czyż, Acta Phys. Pol. B44 (2013) 2281.

[14] M. Benayoun, P. David, L. DelBuono, F. Jegerlehner, Eur. Phys. J. C73 (2013) 2453 [arXiv:1210.7184]. 